\title{
EL CHOQUE CULTURAL ACADÉMICO DEL ALUMNADO ESTADOUNIDENSE EN LA UNIVERSIDAD ESPAÑOLA
}

\section{THE ACADEMIC CULTURE SHOCK OF AMERICAN STUDENTS IN THE SPANISH UNIVERSITY}

\author{
SUSANA PASTOR CESTEROS \\ Universidad de Alicante \\ spc@ua.es
}

\author{
JILLIAN M. PANDOR \\ Curtin University \\ jillian.pandor@curtindubai.ac.ae
}

\section{RESUMEN}

El proceso de internacionalización de los actuales estudios universitarios ha propiciado un número cada vez mayor de alumnado extranjero en las universidades de habla hispana que cursa su Grado o Posgrado en español como segunda lengua. Este fenómeno ofrece muchos ángulos de interés. Entre ellos, el presente artículo tiene como objetivo determinar si el alumnado estadounidense que se integra en el sistema universitario español a través de un programa de Study Abroad experimenta algún tipo de choque cultural académico, así como averiguar su magnitud y características, en caso de que así sea. Para ello, hemos utilizado una metodología cualitativa mediante el diseño de un cuestionario que fue diseminado entre las cinco sedes del programa de Study Abroad del ámbito español (Madrid, Alicante, Barcelona, Palma de Mallorca y Sevilla). El estudio se ha realizado durante el curso académico 2015-2016 y el número de participantes ha sido de 95 estudiantes. Los resultados muestran la existencia de un evidente choque cultural académico, que describimos con detalle, causado por las diferencias del sistema universitario respecto a la cultura académica española y la falta de conocimientos previos sobre ella. Confiamos en que investigaciones como esta contribuyan a paliarlo y se establecen las futuras líneas de trabajo que pueden seguirse para avanzar en este ámbito.

Palabras clave: Choque académico, español académico L2, Study Abroad, integración académica, multiculturalidad. 


\section{ABSTRACT}

The process of internationalization of university studies has contributed to a consistent increase in the number of foreign students currently enrolled in undergraduate and postgraduate studies in Spanish speaking universities where the target language in the classroom (ie. Spanish) is the second language of the foreign student body. This phenomenon lends itself to many different interesting research angles, one of which is explored in this article. Throughout this work our objective is to determine if American students who directly enroll in the Spanish university system through a Study Abroad program experience some type of academic culture shock, and if so, in what way is this experienced and to what degree. In order to achieve this objective we employed qualitative methodology to design a survey that was then sent to all five offices of a Study Abroad program located in Spain (in Madrid, Alicante, Barcelona, Palma de Mallorca and Seville). The study took place during the 2015-2016 academic year with a total number of 95 participants. The results proved that there is a significant "clash" in academic culture (which we discuss in detail throughout this article) caused by the differences that the Spanish academic culture and university system present to this group of students, as well as a general lack of prior knowledge in this regard on the part of the American student body. We believe that this type of research will not only help to alleviate these issues, but also create a clear path for future research that will inevitably continue to contribute advancements to this practically unexplored territory.

Keywords: Clash in academic culture, Academic Spanish L2, Study Abroad, academic integration, multiculturality.

Recibido: 01.09.2017. Aceptado: 10.11.2017.

\section{INTRODUCCIÓN}

- $\mathrm{n}$ el contexto de la actual internacionalización de los estudios universitarios, es Cada vez más frecuente encontrar en las universidades de habla hispana alumnado extranjero que cursa su Grado o Posgrado en español, la que para ellos es una lengua adicional, no la propia, con lo que ello implica de cara al aprendizaje tanto de la lengua como del contenido impartido. Recibir instrucción en una segunda lengua como idioma vehicular es un fenómeno cada vez más extendido, que ha dado lugar a una incesante bibliografía, especialmente en el campo del inglés académico dedicado a estudiantes extranjeros (Reinders, Moore y Lewis, 2008; Reinders, Lewis y Phung, 2017). Sin embargo, en el ámbito del español como segunda lengua (L2, en adelante), a pesar del mencionado cambio del perfil de los estudiantes en nuestras universidades, queda aún mucho espacio para investigar, tal como se explica a continuación. 
Ciertamente, existe un creciente interés por el estudio del español académico, sobre todo desde la perspectiva de la lengua materna (L1, en adelante) (Ballano y Muñoz, 2015; Bolívar y Parodi, 2015; Castelló, 2015; Bazerman et al., 2016; Jiménez y López, 2017; Navarro et al., 2016; Regueiro y Sáez, 2013). También se han venido desarrollando trabajos en español académico como L2, desde la línea iniciada por el proyecto ADIEU (Akademischer Diskurs in der Europäischen Union) dirigido por Vázquez, que abarca tanto la dimensión práctica en el aula (Vázquez, 2001a, 2001b, 2001c, 2001d, 2001e) como la posterior reflexión teórica sobre las implicaciones de la comunicación académica (Vázquez, 2004, 2005, 2009). Con posterioridad, se ha seguido trabajando en aspectos más concretos como la tipología textual académica (Ainciburu, 2012, 2013; Ainciburu y Rodríguez, 2015), la certificación del español académico (Mendoza, 2015) o la internacionalización de la universidad española desde la perspectiva tanto del alumnado extranjero como del profesorado que le imparte docencia (Pastor Cesteros, 2015a, 2015b, 2016, Pastor Cesteros y Rodríguez-Lifante, 2017). Sin embargo, existe un espacio todavía sin explorar a fondo, que es el referido a la adaptación por parte del estudiante extranjero a la cultura académica de la universidad de acogida, para conocer así cuáles son sus necesidades (Pastor Cesteros, 2006). Ese es el tema que en este trabajo presentamos, que tiene como objetivo abordar la multiculturalidad de las aulas desde el punto de vista del alumnado internacional acogido, en particular por lo que se refiere al de origen estadounidense. Es decir, cómo se integra académicamente, si existe o no lo que se ha dado en llamar choque académico cultural para, en caso afirmativo, definir en qué consiste y explorar cómo se puede paliar.

El colectivo de estudiantes norteamericanos en la universidad española tiene una gran tradición y una interesante trayectoria, cuya especificidad no ha sido profusamente estudiada, aunque existen por supuesto trabajos que abordan las dificultades que pueden llegar a experimentar (González, 2004). Por lo que respecta a la situación actual, según la publicación más reciente del informe Open Doors 2016 del Institute of International Education (IIE), durante el año escolar 2014-2015 hubo 313.415 estudiantes estadounidenses que cursaron asignaturas universitarias oficiales durante un programa de Study Abroad, la mayor cifra que se ha visto nunca (IIE, 2016). En ese contexto, alrededor de un 11\% decidió cursar específicamente la modalidad de Direct Enroll o matrícula directa (a través de la que se estudia una materia junto a los alumnos propios de una universidad extranjera). Como es conocido, dentro de cualquier programa de Study Abroad, se pueden mejorar las competencias lingüísticas, así como enriquecer las culturales. Sin embargo, muchas veces, el hecho de vivir con un nativo (como en un homestay) mientras se cursa el programa de Study Abroad o simplemente el estar en un entorno donde se habla el idioma meta, no es suficiente como para conseguir estos objetivos (Kinginger, Wu, Lee y Tan, 2016; Abad, 2008). En cambio, la si- 
tuación de Direct Enroll tiene la particularidad de que aporta un input constante, auténtico y de alta calidad (Rueda, 2006). Un alumno de matrícula directa tiene garantizada la exposición a un registro lingüístico formal con el que de otro modo es difícil que entre en contacto, ya que cursa clases para nativos, impartidas por profesores que tienen que hablar al mismo nivel para todos, tanto los nativos como los no nativos, a diferencia de lo que sucede en un aula de idiomas (o de cualquier materia, pero para alumnado específicamente extranjero), donde son abundantes las modificaciones lingüísticas y conversacionales del profesor, que busca ser entendido por sus estudiantes (González, 2001; Bonilla, 2015). Otra de las ventajas de la opción de Direct Enroll es que en ella se oferta una mayor variedad de cursos, posibilitando así una práctica continuada y significativa en la L2 vehicular, dentro de los principios del aprendizaje integrado de contenidos y lenguas extranjeras (Pastor Cesteros, 2014); por último, permite también la adquisición de un discurso de especialidad, algo crucial para quienes piensan utilizar sus destrezas lingüísticas en un sector profesional concreto en el futuro, una vez finalizados los estudios.

Independientemente de la modalidad elegida, si indagamos sobre las preferencias de destino del colectivo de estadounidenses anteriormente citado, y siguiendo el informe de Open Doors 2016 que mencionamos, vemos que "Europa sigue siendo uno de los destinos más populares para los alumnos estadounidenses que estudian en el extranjero, con un tercio de todos los alumnos de intercambio que van al Reino Unido, Francia y España" (traducción propia) (IIE, 2016, p. 15). Se puede inferir que influye en tal decisión el idioma oficial de cada país en cuestión. Ahora bien, también es cierto que existen otros muchos países de habla hispana aparte de España. Si se observa más detenidamente los datos de Open Doors 2016, se aprecia que de los 170.879 alumnos estadounidenses que cursaron programas de Study Abroad en Europa durante el año académico 2014-2015, 28.325 de ellos estudiaron en España. De ellos, el 65\% son mujeres y un 35\% hombres (PérezBedmar, 2015). Si se compara esta cifra con las correspondientes a los países de América Latina durante el mismo periodo, se observa que ninguno de ellos acogió ni a la mitad de estudiantes. Así pues, se confirma la mayor presencia de estudiantado estadounidense en España frente al resto de países hispanoparlantes. Ello justifica también su elección en este estudio como colectivo de informantes. Se puede deducir que las cifras de alumnado que realiza su Study Abroad en España no hará sino aumentar, por el pujante auge que está cobrando el español a nivel internacional, por supuesto, pero en particular en la vida diaria de los Estados Unidos, se sea o no hablante del idioma (Lacorte y Suárez, 2014).

Ante una situación como la que se ha intentado describir brevemente, es comprensible que un alumno opte por estudiar en España durante su semestre en el extranjero, ya que no cabe duda de que un riguroso programa de Study Abroad facilita la competencia lingüística y la fluidez que pueda llegar a alcanzar 
en español. Esta noción ha sido confirmada por muchos estudios en el campo (Segalowitz et al., 2004), según atestiguan los numerosos artículos de investigación publicados en la revistas específicamente dedicadas a este tema Study Abroad Research in Second Language Acquisition and International Education o Journal of Immersion and Content-Based Language Education.

Ello no implica que todos los participantes sean de carreras relacionadas con la Filología Española (muchos solamente cursan un Minor [especialización en ella] y otros simplemente tienen interés por la lengua o la cultura). No obstante, sí es cierto que la gran mayoría pretende adquirir los niveles lingüísticos necesarios para su futura carrera profesional relacionada de un modo u otro con el español. El rigor del programa de Study Abroad escogido tiene sin duda una correlación directa con el nivel de mejoras lingüísticas experimentadas, y ya hemos comentado que el mayor rendimiento que se puede conseguir durante una estancia en el extranjero es el que se experimenta a través de la modalidad de matrícula directa. Es por ello por lo que suponemos que los alumnos de Direct Enroll eligen esta opción: porque constituye un gran reto cognitivo, académico y personal para ellos y consecuentemente, les brinda la oportunidad de conseguir grandes logros en cuanto a sus competencias lingüísticas y profesionales. En la medida en que una parte medular de estas asignaturas viene determinada por la clases magistrales impartidas por el profesorado, hemos tenido también muy en cuenta la bibliografía existente sobre esta cuestión, tanto de manera general (Morell, 2004, 2009) como referida al español en particular (Ortega, 2001).

Sin embargo, como esta modalidad no siempre se considera la usual, ni la más popular, debido al gran esfuerzo que supone por parte del alumnado, lo cierto es que muchas veces este no está lo suficientemente preparado para ello, lo cual merma las posibilidades que de otro modo podría brindarle la experiencia. Si tenemos en cuenta los requisitos de entrada tan exigentes que se necesitan para cursar programas que ofrecen esta modalidad, podemos afirmar que el alumnado que participa en ella parte con la mejor competencia lingüística y trayectoria académica. Como se trata de un colectivo muy capacitado, consideramos que la aportación de los recursos necesarios en concepto de preparación y apoyo es algo que puede impulsarlos a conseguir un mayor rendimiento académico de su estancia. Fomentar esta opción adecuadamente haría evolucionar la visión actual de Study Abroad. Se transformaría de una experiencia creada por una organización externa y hecha a medida para los participantes de una nacionalidad en concreto, a una vivencia académica que cultiva estudiantes del mundo, realmente internacionales y multiculturales, que les aporta una nueva mentalidad global, así como mejoras lingüísticas, culturales y personales, tras vivir y disfrutar de todo lo que aporta el poder desenvolverse en un curso oficial en la universidad de acogida (Cohen, Paige, Kappler, Chi y Lassegard, 2005).

Este es el contexto en el que hemos desarrollado este trabajo, que estructu- 
ramos del siguiente modo. Tras esta introducción, presentamos las preguntas de investigación que se nos plantean y, a raíz de ellas, los objetivos que perseguimos. Mostramos a continuación la metodología que hemos seguido y cómo se ha diseñado la investigación, para analizar y discutir seguidamente los resultados obtenidos. Finalmente, cerramos el estudio con las conclusiones que pueden derivarse de él y esbozamos algunas de las futuras líneas de trabajo con las que esperamos que pueda tener continuidad.

\section{PREGUNTAS DE INVESTIGACIÓN Y OBJETIVOS}

Tras revisar la bibliografía existente y a partir de nuestra propia experiencia sobre el tema, hemos planteado una serie de preguntas, que presentamos a continuación, que son las que han guiado el presente trabajo de investigación. De las conexiones entre ellas se deriva a su vez nuestro objetivo principal.

Nos interesaba averiguar exactamente el tipo de asignaturas que causa dificultades al alumnado estadounidense de intercambio, con el fin de establecer qué es más problemático, la materia o la metodología. De ahí surge nuestra primera pregunta:

- Entre el colectivo de alumnado estadounidense de matrícula directa que estudia en una universidad española, ¿cuáles son las asignaturas que mayoritariamente están cursando?

Por otro lado, aunque disponer de información sobre la materia impartida es de gran relevancia, hace falta conocer también el idioma en que se imparte, porque es sabido que cada vez más este alumnado se acoge a la creciente oferta de asignaturas en inglés en las universidades españolas. Esta información nos permitirá contrastar las experiencias del colectivo estudiantil según la lengua vehicular de la asignatura. Es por eso por lo que nos preguntamos:

- De las asignaturas cursadas ¿cuántas se imparten en español? Y ¿cuántas en inglés?

Con el fin de comparar las culturas educativas estadounidense y española, para poder juzgar así el alcance de las diferencias que existen entre ambas, así como la gravedad del choque que puede llegar a experimentar el alumnado estadounidense, partimos de las cuestiones planteadas por Pastor Cesteros (2006) y establecimos nuestra última pregunta de investigación: 
- ¿Cuáles son las dificultades que experimentan los alumnos estadounidenses en cuanto a la cultura académica española, en particular en lo referido a la comprensión e interacción en la clase magistral?

En definitiva, gracias a las respuestas a las tres preguntas anteriores, sabremos cuáles son las carencias que actualmente existen en la preparación de este alumnado para estudiar en español L2, así como la información más relevante en este sentido, a partir de sus propias experiencias.

Consecuentemente, el objetivo principal de este trabajo consiste en determinar si el alumnado estadounidense que se integra en el sistema universitario español a través de un programa de Study Abroad experimenta algún tipo de choque cultural académico, así como averiguar su magnitud, en caso de que así sea. Para ello, indagamos en la relación que se establece entre el estudiante visitante y su nueva universidad de acogida en el extranjero. Este objetivo está directamente relacionado con las tres preguntas de investigación anteriores, a través de las que pretendemos averiguar las dificultades experimentadas por este colectivo estadounidense con respecto a la materia impartida o durante la clase magistral en español. Para responderlas, hemos recabado información de naturaleza subjetiva que se basa en las percepciones personales del alumnado, como explicamos en el siguiente apartado de metodología, por lo que anticipamos muchísima variedad entre los datos conseguidos.

Nuestra hipótesis de partida era que la falta de preparación del alumnado con respecto a las muchísimas diferencias que existen en cuanto a la cultura académica cuando un alumno estadounidense se incorpora a una asignatura de una universidad española con profesorado y alumnado nativos, de la que además ha de evaluarse, puede llegar a empeorar la experiencia. A su vez, pensamos que estas diferencias seguirán siendo un asunto problemático incluso con independencia del idioma en que se imparta la clase (es decir, tanto en español como en inglés), ya que creemos que están relacionadas más con la metodología universitaria empleada que con la falta de suficiente competencia lingüística por parte de este colectivo estudiantil. Por todo ello, creemos que el alumnado estadounidense de cualquier programa de Study Abroad con experiencia de Direct Enroll, para un mejor rendimiento académico, requiere formación previa en cuanto a la cultura académica del país de destino y las diferencias que inherentemente presenta en comparación con la suya de origen.

En el apartado 4 veremos hasta qué punto se ha verificado o refutado esta hipótesis. Previamente, a continuación, explicamos la metodología empleada y el diseño de la investigación realizada. 


\section{METODOLOGÍA Y DISEÑO DE LA INVESTIGACIÓN}

Puesto que el estudio que presentamos gira en torno a las creencias, percepciones y opiniones personales del alumnado que ha experimentado una estancia de matrícula directa en una universidad española, decidimos que la metodología cualitativa sería sin duda la más apropiada para llevar a cabo nuestra investigación. Es por eso por lo que diseñamos un cuestionario para distribuirlo en forma de encuesta en línea. Ello facilitó difundirlo ampliamente y así fomentar la participación de estudiantes que se encontraban en cinco ciudades distintas del ámbito nacional español (correspondientes a las cinco sedes del programa de Study Abroad en cuestión: Madrid, Alicante, Barcelona, Palma de Mallorca y Sevilla).

La fecha de diseminación de la encuesta fue el curso académico 2015-2016. Ahora bien, al realizar la misma encuesta dos veces para el alumnado de ambos semestres (otoño y primavera), las enviamos al final de cada uno de ellos, para así obtener una visión más completa de la experiencia de cada colectivo estudiantil. Para el primero, solicitamos participación durante dos semanas, desde el 7 hasta el 18 de diciembre de 2015. Para el segundo, desde el 16 hasta el 31 de mayo de 2016.

Finalmente, el número de informantes de esta investigación fue de 95 estudiantes de la modalidad de matrícula directa a lo largo de los dos semestres citados.

Respecto a la encuesta, decidimos crearla a través de LimeSurvey.org, puesto que este sitio web está especializado en la elaboración de cuestionarios y ello nos permitía diseñar el nuestro de forma sencilla, pero a su vez sofisticada, gracias a su gran variedad de formatos de preguntas. La creamos de modo que, según la información aportada por el alumno, y donde fuera relevante, el programa automáticamente le ofreciera otro ítem específico a continuación. Así, los participantes solamente contestaron a cuestiones que tenían que ver con sus anteriores respuestas. Por ello, no podemos ofrecer un número exacto correspondiente a la totalidad de preguntas incluidas en la encuesta, porque en realidad no tenía un único formato; no obstante, la media de preguntas contestadas por los participantes ronda las 37.

La encuesta estaba compuesta por una introducción y cinco apartados de preguntas. Una vez creada, hicimos un pilotaje con cinco estudiantes que previamente habían cursado un programa de Direct Enroll e implementamos pequeños cambios a partir de su retroalimentación. En su versión final, las cinco secciones de la encuesta giraban en torno a los siguientes aspectos: el perfil del participante, la información general sobre el programa de Study Abroad cursado, la comparación entre la universidad española y la estadounidense, la preparación recibida y las impresiones de las clases de matrícula directa en la universidad española.

En la sección del "perfil del participante", preguntamos acerca de su edad, sexo, universidad de origen, curso académico y carrera universitaria. También les solicitamos que autoevaluaran su nivel de español antes de empezar su semestre 
en el extranjero (con respecto a cada una de las destrezas y al léxico). Este ítem de autoevaluación está basado en los niveles establecidos por las directrices del American Council on the Teaching of Foreign Languages (ACTFL, 2012), porque son las que se usan globalmente en los EE. UU. y pensamos que la terminología para cada nivel sería más fácilmente identificable por los participantes que la escala del Marco Común Europeo de Referencia para la Lenguas (MCERL, 2002). Por otro lado, para justificar la validez de la autoevaluación, nos basamos en Griffin (2007, p. 115), quien asegura que, a pesar de que las técnicas introspectivas muchas veces son criticadas por una supuesta falta de objetividad, y por lo tanto de fiabilidad metodológica, lo cierto es que la imagen mental es fundamental para la configuración de lo que los aprendices consideran un hablante nativo "ideal". Por lo tanto, su autoevaluación permite la identificación del propio nivel de una manera válida para los propósitos de nuestro trabajo. Con toda esta información, se pretende averiguar cuántos estudiantes cursaban una carrera relacionada con la lingüística española y cuántos cualquier otra disciplina, así como las tendencias que se pudieran generar a lo largo de la encuesta entre alumnado del mismo sexo, edad o incluso nivel lingüístico. Cabe destacar, finalmente, que todos los datos recopilados a través de la encuesta, en esta sección y en todas las demás, se han aportado de forma anónima, sin pedir en ningún momento los nombres y apellidos de los participantes.

En el segundo apartado, sobre "información general acerca del programa de Study Abroad", se preguntó el nombre del programa de Study Abroad en el que estaban inscritos, si se trataba de un semestre o del año académico entero, la ciudad y la universidad de destino en España a través de la que realizaron su matrícula directa, el número de asignaturas que estaban cursando en ella, tanto en español como en inglés, los títulos de las asignaturas, si su nota final de las clases de Direct Enroll se transferían a su universidad de origen o si se trataba solo de una transferencia de créditos del tipo "aprobado/suspenso" y, en último lugar, si en el momento de ejecutar la encuesta habían realizado ya algún tipo de tarea o evaluación para su(s) asignatura(s) de matrícula directa que contara para su nota. A través de esta sección se utilizó la opción ya mencionada de establecer preguntas específicas derivadas de las respuestas previamente aportadas, puesto que, para aumentar la fiabilidad de la encuesta, queríamos que solamente un alumno de una sede de Study Abroad en concreto pudiera contestar preguntas relacionadas con ella.

Este segundo apartado sirvió para identificar a los alumnos que provenían de cada sede del programa de Study Abroad para después contrastar sus respuestas con la preparación que hubieran recibido y la experiencia en general. A su vez, permitía la opción de comparar las dificultades que experimenta el alumnado de una universidad española en concreto con las de otra, así como los inconvenientes de los estudiantes que cursan clases de matrícula directa en inglés con los del colectivo que las recibe en español. Asimismo, facilitaba la potencial identificación de 
asignaturas o materias que pudieran ser de mayor dificultad. Las preguntas finales concedían una visión más afinada de la experiencia del estudiante, así como su perspectiva y actitud con respecto a ella en general. La cuestión de la transferencia de notas de la universidad española a la de origen es crucial, porque indica un mayor o menor nivel de preocupación por parte del alumnado en cuanto a sus clases universitarias en el extranjero. Si algunas de sus notas o todas ellas se transfieren de forma directa, ello implica que tienen un gran impacto en el promedio de sus calificaciones y, por tanto, de su expediente académico (lo cual no solo constituye un requisito para graduarse sino, sobre todo, un elemento competitivo a la hora de conseguir empleo), por lo que se puede suponer un mayor nivel de estrés y ansiedad a la hora de querer rendir al máximo académicamente. Por el contrario, en caso de tener solamente que aprobar todas las asignaturas de Direct Enroll para poder recibir los créditos correspondientes, se presume que el alumno se enfrentará a la experiencia en la universidad de destino de forma mucho más relajada. Finalmente, la última pregunta tiene una incidencia directa en las respuestas a las cuestiones que aparecerán en los apartados subsecuentes, ya que indica si los alumnos han sido evaluados ya o no. La respuesta afirmativa a este ítem indica una visión más completa de la experiencia de matrícula directa en su totalidad, evidentemente porque incluye el componente de evaluación. En caso de una respuesta negativa, a la hora de analizar los datos, se ha tenido en cuenta que el alumno en cuestión solamente posee una percepción parcial de lo que consiste el proceso de Direct Enroll, ya que es posible que no haya recibido ningún tipo de retroalimentación, que no sepa nada sobre su propio progreso, ni haya experimentado de primera mano los criterios de evaluación que aplica el docente.

La tercera sección de "comparación entre la universidad española y la universidad estadounidense" planteaba tres preguntas (con una escala de Likert de tres opciones) a través de las que el alumnado tenía que identificar su nivel de acuerdo o desacuerdo con el enunciado en cuestión. El primero pretendía identificar el nivel general de diferencias percibidas entre la universidad española y la estadounidense en cuanto a la metodología docente (el modo de dar clases). El segundo componente consistía en analizar 18 aspectos relacionados con la clase magistral y señalar si se dan en la universidad española más, menos o igual que en la universidad estadounidense. El último ítem dentro de esta sección constaba de un duplicado del listado de los 18 aspectos de la pregunta anterior, esta vez ya marcados con las palabras "más / menos que en los EE. UU.", de manera que, entre ellos, tenían que elegir los tres aspectos diferentes de la cultura educativa en España que constituían las mayores causas de dificultad para ellos. El objetivo es obvio: observar la coincidencia entre los participantes a la hora de identificar los aspectos que pudieran determinar el choque académico cultural experimentado al incorporarse a la universidad española.

La penúltima sección, titulada "Preparación", consistía en conocer el apoyo 
aportado de antemano por su universidad de origen en los EE. UU., el que les fue ofrecido por su programa de Study Abroad antes de que comenzara el semestre, sus conocimientos acerca de los servicios de asistencia académica que ofrece el programa de Study Abroad a lo largo del semestre en cuanto a sus clases de matrícula directa y si los habían utilizado o no. En caso de usarlos, se indagó en si les resultaron útiles y en caso contrario, se preguntó por qué no los utilizaron. A su vez, se les preguntó acerca del apoyo que recabaron de sus profesores en la universidad, así como su valoración del mismo. Ello permitía, a su vez, apreciar cómo perciben al docente universitario a la hora de solicitar ayuda.

Por último, el apartado sobre "las impresiones de las clases de matrícula directa en la universidad española" demandaba una reflexión general sobre la experiencia en la universidad de acogida y su satisfacción con la misma. Se preguntó si recomendarían la modalidad de Direct Enroll a un amigo que quisiera estudiar en España y por qué o por qué no. Se indagó acerca de las mejoras lingüísticas que atribuían a su experiencia de matrícula directa y en qué destrezas del idioma lo habían observado más. Para cerrar el círculo, se les pidió una valoración de su nivel de español tras su semestre de Direct Enroll, en el mismo formato del primer apartado. Las dos preguntas finales de esta sección eran de respuesta abierta. Una sobre qué era lo que les habría gustado saber acerca de la experiencia de matrícula directa en la universidad española antes de llegar a España, y la otra era simplemente para cualquier comentario adicional que consideraran pertinente.

Para la diseminación de la encuesta pedimos la ayuda de los coordinadores del programa de Study Abroad, ya que no les estaba permitido aportarnos la información de contacto de sus alumnos. Su colaboración a la hora de difundir el cuestionario en línea y animar a la participación en ella a través de sus clases presenciales en su centro de estudio ha sido esencial para la satisfactoria realización de este estudio.

\section{ANÁLISIS Y DISCUSIÓN DE RESULTADOS}

A pesar de que, como puede deducirse del contenido de la encuesta explicada en el apartado anterior, los datos obtenidos fueron muy amplios y de diversa temática, para los objetivos de este artículo nos centraremos en analizar y comentar los resultados acerca del perfil de los participantes y, especialmente, los que tienen que ver con la comparación entre la cultura académica de los dos sistemas universitarios (español y estadounidense) y con las impresiones de los estudiantes sobre su experiencia de matrícula directa. Aquí se discutirán en detalle y se destacarán los más relevantes para el tema que nos ocupa.

Así pues, en primer lugar, y por lo que se refiere al perfil de los participantes, tomando la media de los datos de los semestres de otoño 2015 y primavera 2016, 
se observa que, de los 95 participantes, el $81 \%$ eran mujeres ( 77 participantes en total), mientras que el 18,95\% eran hombres (18 en total) (Gráfico 1). La edad media es de 20,5 años, con un $80 \%$ de los alumnos (76 de ellos) que se encuentran en estos momentos cursando su tercer año de carrera o junior year, como se denomina en los EE. UU.

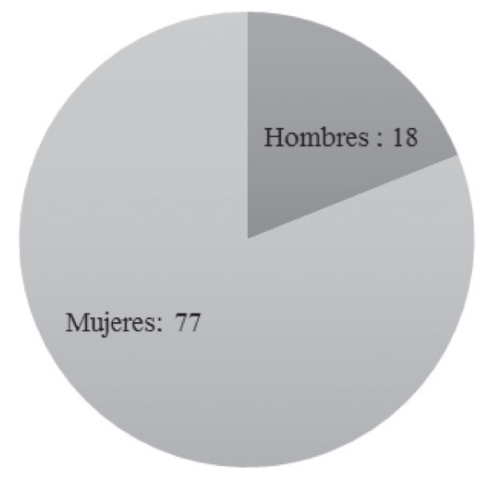

Gráfico 1. Resumen de datos obtenidos en la sección de "Perfil del participante".

Respecto al número de carreras representadas en total (incluidas en el Gráfico 2), habiendo eliminado las repeticiones entre semestres, que nuestros participantes cursan 53 carreras distintas en las diversas universidades estadounidenses. Como prueba del 'compromiso ético' con el trabajo que poseen muchos de estos alumnos, en total había tres estudiantes que estaban cursando una triple carrera y 45 una doble carrera, lo cual quiere decir que el 50,53\% de la población encuestada (48 participantes) estaba cursando más de una carrera universitaria en su institución de origen en los EE.UU.

Si se pone atención en la materia en sí que estudian, las tendencias en cuanto a la popularidad de las carreras demuestran que el $43,16 \%$ de todos los participantes (es decir, 41 de ellos) está cursando estudios relacionados con la Lengua y cultura españolas. En segundo lugar, están las carreras de Humanidades, seguidas por el 32,63\% de los alumnos (31 en total). Estas incluyen (por orden de popularidad) Psicología, Historia, Sociología y Comunicación, entre otras. En tercer lugar, están las relacionadas con Ciencias Empresariales, que cursa el 27,37\% de la población encuestada (es decir, 26 estudiantes). No obstante, sigue habiendo una buena representación (el 16,84\%) de carreras que a priori se considerarían menos convencionales para un programa de Study Abroad en España, como Matemáticas, Ciencias o algún tipo de Ingeniería (un total de 16 participantes), así como un $3,16 \%$ (tres alumnos) que estudiaba Medicina. Todo ello se puede ver resumido en el siguiente Gráfico 2: 


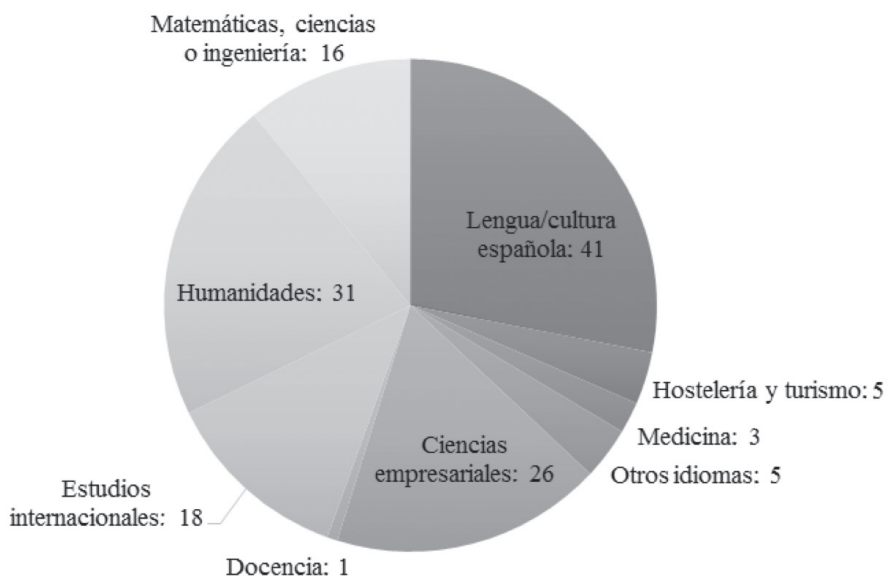

Gráfico 2. Carreras universitarias cursadas por la población estudiantil encuestada.

De los datos recopilados, también resulta significativo que la mayoría (el $61,05 \%)$ de los encuestados cursa sus asignaturas solo en español. En cambio, solamente un 23,16\% de ellos afirma no cursar ninguna asignatura en español, sino en inglés. Entre los que están matriculados en materias impartidas en español, el $27,37 \%$ (el mayor porcentaje que se obtuvo) asiste a tres asignaturas a la vez. Por el contrario, curiosamente, la mayoría de los que cursan asignaturas en inglés (un $24,21 \%$ de los alumnos), solamente siguen una asignatura en la universidad española de acogida durante su estancia en el extranjero.

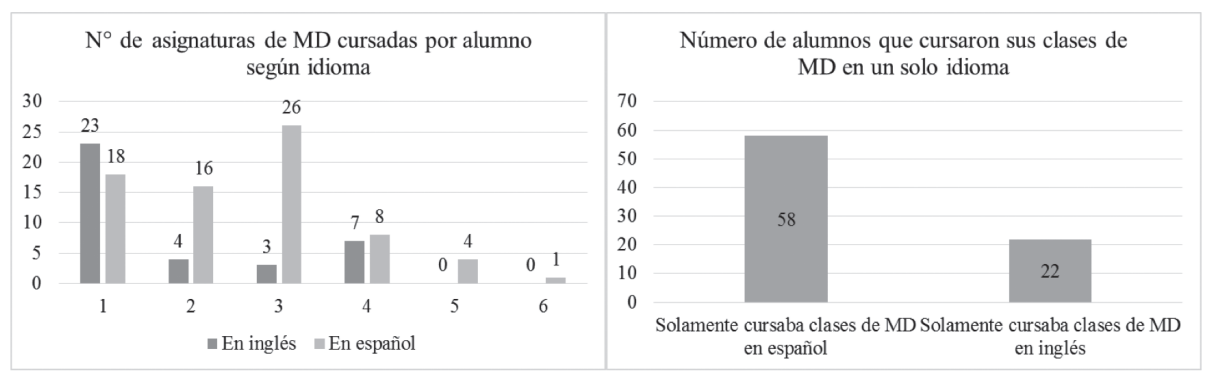

Gráfico 3. Número de asignaturas de matrícula directa cursadas por alumno según el idioma vehicular.

Con respecto a la transferencia de notas y el hecho de haber pasado o no por una fase evaluativa en el momento de rellenar la encuesta, resulta muy interesan- 
te comprobar que nuestros participantes están divididos casi exactamente por la mitad, con el 48,42\% que declara que sus notas se transfieren de forma directa, mientras que un porcentaje muy similar, el 49,47\%, afirma que sus asignaturas solamente cuentan por créditos, es decir, que si aprueban la asignatura, su universidad de origen les concede los créditos correspondientes, sin especificar calificación. El otro 2,11\% de los encuestados menciona que, para algunas asignaturas, el valor de la nota se transfiere a su universidad de los EE. UU., mientras que para otras solamente cuentan los créditos.

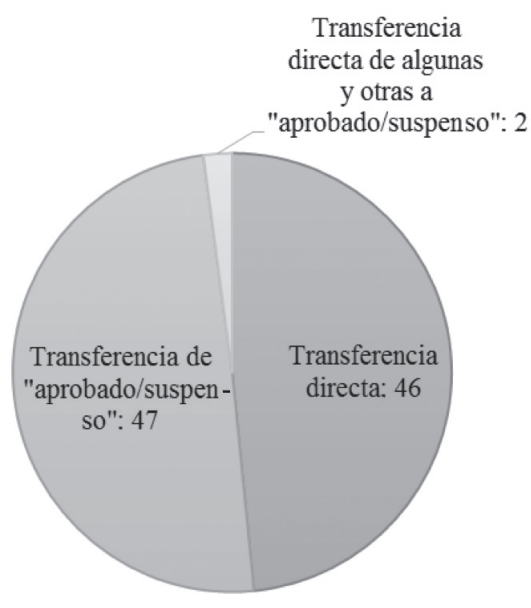

Gráfico 4. Tasa de transferencia de notas a la universidad de origen en los EE. UU.

Curiosamente, más adelante se observó en los resultados de la encuesta que el $68,75 \%$ del alumnado que tenía transferencia de notas recomendaría la modalidad, frente al 73,33\% de los que no tenían transferencia de notas que la recomendaría. Por tanto, concluimos que la transferencia de notas, contrariamente a lo que suponíamos, no ha sido un factor que haya impedido tener una impresión favorable del programa.

No obstante, en sus opiniones sobre la experiencia, creemos que más importante que la transferencia de la nota está el hecho de si habían sido ya evaluados o no en el momento de rellenar la encuesta. Solo si ha pasado por algún tipo de evaluación, el alumnado poseerá una visión completa sobre lo que es pasar un semestre en una universidad española, ya que los exámenes se suelen dar al final del semestre y ocupan un gran porcentaje de la nota. Así pues, según los datos obtenidos, el $84,21 \%$ de nuestros estudiantes habían pasado por la fase de evalua- 
ción, frente a solo el 15,79\% que en el momento de responder no la había experimentado todavía (Gráfico 5). Igual que para las notas, se utilizó esta información para ver cómo cambian las percepciones del alumnado cuando se comparan las valoraciones de la experiencia de los que habían sido evaluados frente a los que no y se descubrió, tras analizar sus otras respuestas, que el $80 \%$ de ellos no tenía transferencia de notas, lo cual probablemente indica que no estaban tan preocupados por dicha evaluación, sino simplemente por aprobar la asignatura.

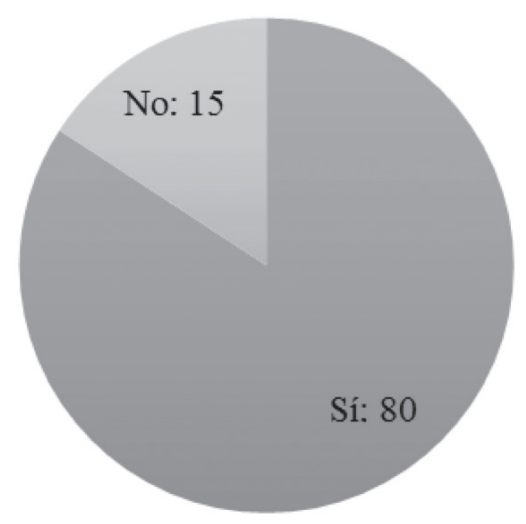

Gráfico 5. Desglose de alumnado que había pasado por alguna fase evaluativa en el momento de rellenar la encuesta.

Nos adentramos ahora en uno de los aspectos medulares del tema, es decir, si los alumnos observaron diferencias en cuanto a la cultura académica al comparar sus experiencias en ambos países. A partir de ahí, si habían advertido cualquier disimilitud, se les preguntó en qué aspectos concretos la cifraban. Finalmente, se les pidió una valoración de dichas diferencias teniendo en cuenta la dificultad que les generaban a la hora de incorporarse al sistema educativo español. Todo ello ha facilitado una mejor comprensión de la perspectiva del alumnado visitante, que ahora se presenta.

El primer ítem consistía en identificar el grado de diferenciación o similitud que percibían entre ambos sistemas académicos, para lo que podían elegir entre cuatro descriptores: "No hay diferencia", "Hay alguna diferencia", "Hay una diferencia significativa" o "Hay una diferencia tremenda". Como se puede ver abajo en el Gráfico 6, el 100\% de los participantes estaba de acuerdo en que la cultura académica entre los dos contextos no es en absoluto igual. 


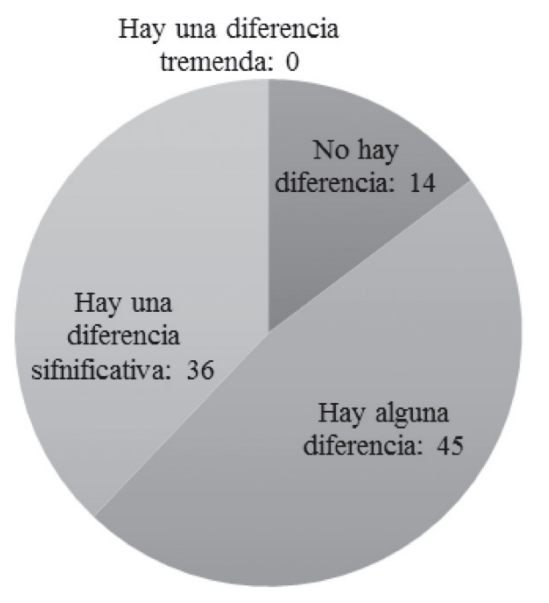

Gráfico 6. Perspectiva del alumnado en cuanto al grado de diferenciación entre ambos sistemas educativos.

Para diagnosticar realmente la situación y poder especificar lo que se podría considerar como un "choque" respecto de la cultura académica, se les proporcionó un listado de posibles diferencias en cuanto al desarrollo de la clase magistral en cada uno de los dos países (el aspecto en el que nos hemos centrado, según justificamos en la introducción) y se les solicitó que indicaran si cada uno de esos aspectos se daba en España más, menos o igual que en los EE. UU.

De todos los resultados, extraemos los siete factores con mayor porcentaje de coincidencia entre los informantes respecto a las diferencias observadas y, consecuentemente, en cuanto a la dificultad que les provocan, tal como se puede observar en el Gráfico 7. Cabe destacar que los números que están representados en el gráfico no coinciden con el número total de participantes de cada semestre, ya que, a través de este ítem, tenían que seleccionar tres respuestas. Los más seleccionados están relacionados directamente con la evaluación, lo que ratifica la importancia de la nota para los estadounidenses y cómo, muchas veces, la consideran más importante que el propio aprendizaje conseguido. Aunque existe mayoría absoluta, el 43,16\% de nuestros participantes indica que el hecho de tener menos oportunidades de evaluación que en sus universidades de origen constituye uno de los principales inconvenientes a la hora de adaptarse al sistema español. A su vez, a un $36,84 \%$ le resulta difícil de entender que al examen final le corresponda tanto 
valor de la nota final de la asignatura. Luego, con menor representación, pero siguiendo con el tema de las calificaciones, vemos que el 28,42\% del alumnado considera que el hecho de tener menos posibilidades de conseguir una "nota alta" es una desventaja, mientras el $25,26 \%$ de los alumnos estima igualmente problemático tanto el menor nivel de retroalimentación aportado por los docentes como el hecho de que estos estuvieran en general menos involucrados en el éxito académico del estudiante. Estas dos cuestiones son muy interesantes y representativas. Por un lado, parece que el alumnado estadounidense está acostumbrado a recibir mayor feedback sobre su evolución académica (comentarios a sus trabajos escritos, valoración posterior de sus presentaciones orales, indicaciones sobre cómo y por dónde continuar el estudio o el enfoque de una materia...). Eso es algo que en la universidad española se está introduciendo poco a poco en los últimos años, a raíz de la metodología derivada del nuevo Espacio Europeo de Educación Superior (con sus pros y sus contras), sin embargo parece que no acaba de llegar al nivel del que suele recibir el alumnado estadounidense.

Directamente relacionado con lo anterior está el hecho de que existe en los EE. UU., una mayor implicación de los docentes en el éxito o fracaso de sus estudiantes, es decir, que su trayectoria, el alcance de su aprendizaje y las calificaciones obtenidas son sentidas por aquellos como parte de su responsabilidad. Esta creencia no es percibida de tal modo entre el profesorado universitario español, que espera más bien que el estudiante sea autónomo y plenamente autorresponsable de sus logros, lo cual implica un pensamiento implícito respecto del funcionamiento del mundo académico no compartido entre el profesorado español y el alumnado estadounidense. En este sentido justamente, un $18,95 \%$ de la muestra señala que ha constituido un inconveniente para ellos el que los profesores tuvieran la expectativa de que el alumnado había de ser más autónomo. En último lugar entre los factores señalados como diferentes, con igual porcentaje que el anterior, vemos que a los estudiantes estadounidenses les causa especial dificultad el que la clase sea más un monólogo del docente que un diálogo interactivo con los alumnos. Sobre esta cuestión, aunque es cierto que en el sistema universitario español estamos por fortuna lejos de la clase magistral (mal)entendida como un discurso monolítico del profesor, en el que nadie puede intervenir, tampoco parece ser, según los datos que revela nuestro estudio, que se ha avanzado totalmente hacia la clase magistral interactiva tal como queda descrita y promovida por Morell $(2004,2009)$, que claramente defendemos. 

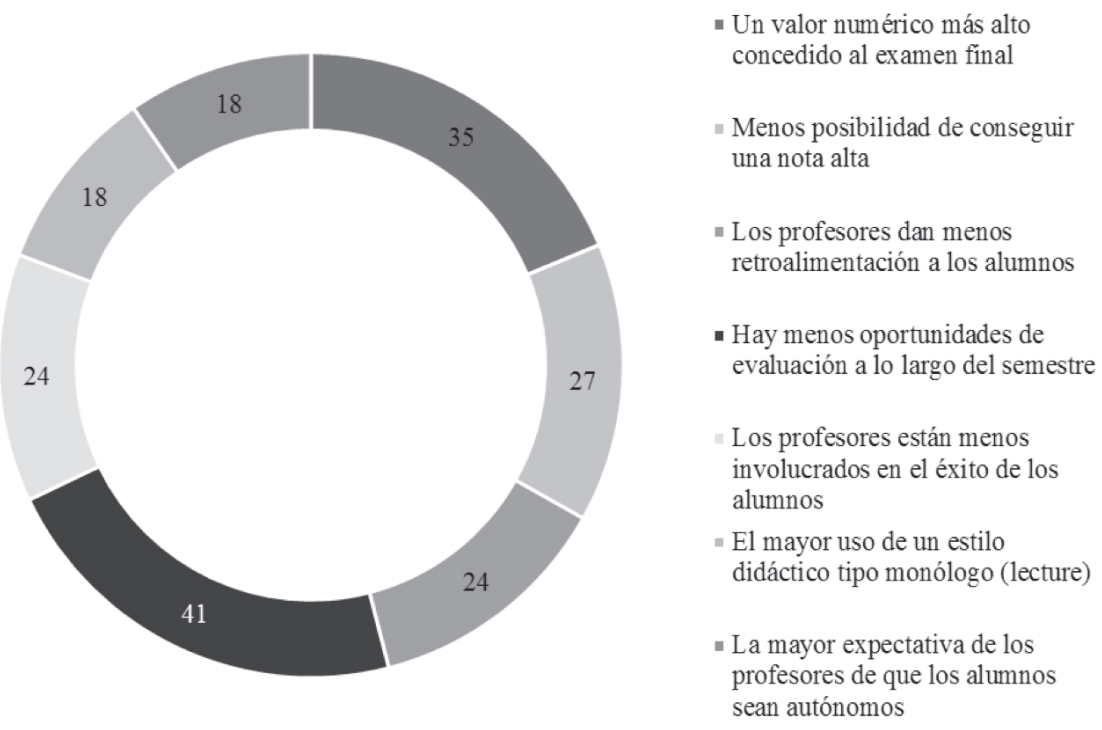

Gráfico 7. Aspectos diferentes entre los dos sistemas académicos que producen mayor nivel de dificultad para los encuestados.

A continuación, con respecto a la cuestión de si realmente la experiencia de matrícula directa les había ayudado a mejorar su nivel de español, se observa que el 67,37\% (la mayoría de los participantes, como puede visualizarse en el Gráfico 8) creen que la estancia, como era de esperar, les ha servido como una manera apropiada de ir más allá de su competencia lingüística de partida.

\section{¿Crees que la experiencia de MA te ha ayudado a mejorar tu español?}

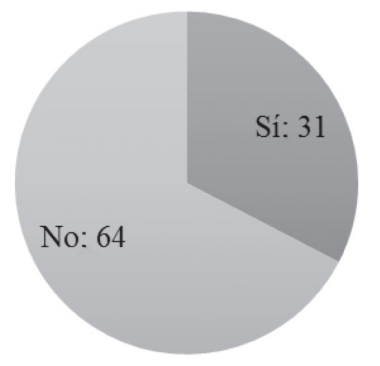

Gráfico 8. Si la experiencia de matrícula directa ha mejorado las destrezas lingüísticas y cómo. 
Esta afirmación viene avalada por la comparación de los datos sobre los niveles lingüísticos que los alumnos indicaron en su autoevaluación al principio del semestre con los de su autoevaluación al final del semestre (Gráfico 9).
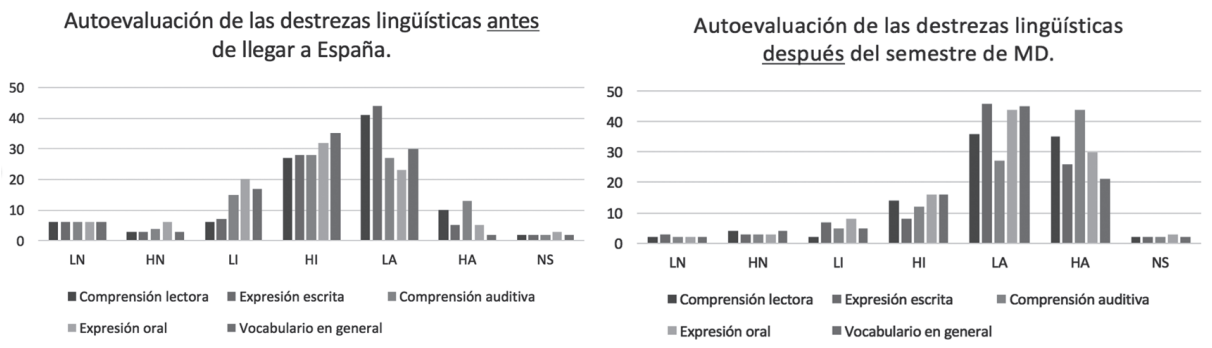

Gráfico 9. Comparación entre la autoevaluación del nivel de español según destrezas antes de llegar a España (izquierda) y después del semestre de matrícula directa (derecha).

Queda claro que, por lo general, en cada destreza hay un mayor porcentaje de alumnado que se sitúa entre los niveles superiores de "avanzado bajo" y "avanzado alto" al final del semestre, lo cual constituye un aumento significativo desde la autoevaluación "antes de llegar a España", cuando la mayoría se situaba en los niveles "intermedio alto" y "avanzado bajo" para cada destreza. Esto nos indica que la mayoría ha aumentado un nivel en cada destreza y en algunos casos, dos. Según los porcentajes que hemos obtenido de cada nivel correspondiente a cada una de las destrezas lingüísticas, los participantes por lo general se han valorado de forma más positiva en esta segunda autoevaluación. Esto implica un aumento no solamente en su competencia comunicativa, sino también en su autoestima y confianza en cuanto al uso del idioma y la percepción de sí mismos como usuarios de la lengua, aspectos ambos muy positivos por la conocida incidencia de la dimensión afectiva en la capacidad de aprendizaje de una segunda lengua y los resultados de la adquisición.

Finalmente, abordamos las percepciones e impresiones del alumnado de manera global acerca de la modalidad de Direct Enroll. Respecto a la pregunta genérica de si recomendarían la opción de matrícula directa a un(a) amigo(a) que viniera a estudiar en España, nos complace comprobar que la gran mayoría de los encuestados (el 69,47\%) responde afirmativamente, lo cual se puede interpretar en el sentido de un alto grado de satisfacción con la experiencia en general, como se puede verificar en el Gráfico 10: 


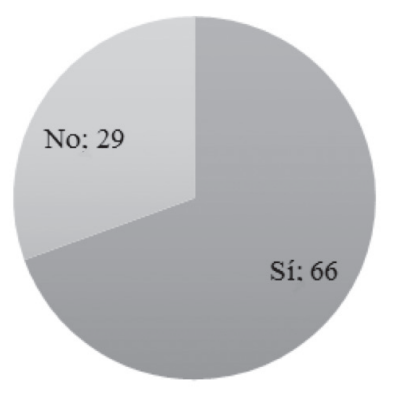

Gráfico 10. Tasas de satisfacción con la modalidad de Direct Enroll.

Tras analizar las justificaciones del 29\% del alumnado que no recomendaría la modalidad, consideramos que los motivos están básicamente relacionados con una falta de expectativas adecuadas de antemano y con problemas concretos con el profesorado a cuyas clases han asistido, aspectos ambos en los que posiblemente podría incidir de una manera positiva la preparación e intermediación de la coordinación académica del propio programa de Study Abroad.

Una vez finalizado el análisis y comentario de los datos de nuestro estudio, que consideramos reveladores en especial por lo que se refiere a la identificación de los aspectos esencialmente diferentes de las culturas académicas española y estadounidense, pasamos a continuación a exponer las conclusiones que podemos extraer de tales resultados.

\section{CONCLUSIONES}

La investigación que se presenta en este trabajo se ha centrado en la que consideramos la opción de Study Abroad más intensiva, fructífera académicamente y gratificante para el alumno, es decir, la modalidad de matrícula directa. Consideramos que un intercambio universitario de estas características rompe con lo que podríamos ejemplificar de forma metafórica como la típica "burbuja" del programa de Study Abroad (en la que el alumnado comparte aula solo con colegas de su misma nacionalidad, en clases adaptadas a su perfil y necesidades específicas) $y$, por el contrario, obliga al estudiante a salir de su zona de confort y adentrarse en un mundo de exploración académica multicultural. Porque integrarse en un curso de una universidad extranjera en una L2 con todas sus consecuencias constituye sin duda un reto personal y académico que reporta a su vez un intenso aprendizaje cultural. Desafortunadamente, muchos alumnos estadounidenses que esperan vivir esta experiencia parten de unas expectativas no realistas, porque creen que poseer un nivel lingüístico adecuado de español es suficiente para matricularse en cualquier asignatura de la universidad española. Sin embargo, como demuestran 
los resultados de este estudio, esta creencia está muy lejos de la realidad y dificulta el que los alumnos de matrícula directa se preparen para superar el choque cultural académico que muchos inevitablemente experimentarán.

Para resumir las principales conclusiones a las que hemos llegado en este sentido, retomamos nuestras preguntas de investigación que comenzaban por intentar cifrar hasta qué punto las dificultades de la experiencia de Direct Enroll estribaban en la materia impartida, en la lengua vehicular de la clase o en las diferencias en cultura académica.

Así pues, respecto a la primera pregunta (¿Cuáles son las asignaturas que están cursando?), tras analizar las detalladas respuestas, podemos deducir que la mayoría de las asignaturas elegidas eran apropiadas y basadas en la carrera del alumno o en sus conocimientos previos, lo cual descarta que esa pudiera ser una fuente de dificultad para ellos. En cuanto a la segunda pregunta, sobre la cuestión medular de la lengua vehicular (De las asignaturas cursadas ¿cuántas se imparten en español? Y ¿cuántas en inglés?), hemos comprobado que la mayoría (el 61,05\%) de los participantes cursó asignaturas exclusivamente en español mientras solamente el $27,37 \%$ lo hizo por completo en inglés. Ello, unido al hecho de que la competencia lingüística de partida era suficiente para interactuar en el ámbito académico y de que recibimos escasas quejas tanto sobre las dificultades de comprensión de la variante dialectal española de los docentes (solo el 5,26\% de la muestra) como sobre el nivel lingüístico del profesorado que impartía docencia en inglés, nos lleva a descartar la lengua vehicular como el principal motivo de dificultad entre el alumnado estadounidense (lo cual no impide que pudiera generar problema, obviamente, pero que no han sido percibidos como fundamentales en esta cuestión del choque académico). En último lugar, por lo que se refiere a nuestra tercera pregunta de investigación (¿Cuáles son las dificultades que experimentan los alumnos estadounidenses en cuanto a la cultura académica española?), los datos obtenidos aportan información muy valiosa sobre la experiencia de este colectivo. Así, las principales dificultades vividas por el contraste del sistema académico de las universidades española y estadounidense estaban relacionadas con los siguientes aspectos: (1) una menor supervisión por parte del profesorado (carecían de la autonomía que aquel esperaba de ellos), (2) el estrés del alto porcentaje que poseía el examen final en la calificación global (con menos oportunidades, por tanto, de una evaluación continua como aquella a la que estaban acostumbrados), (3) la escasa retroalimentación recibida por sus docentes respecto a la evolución de su aprendizaje y (4) las diferencias en la metodología docente, en especial por lo que se refiere a una clase magistral mucho más monológica que interactiva. En definitiva, los participantes especificaron que los factores metodológicos que más contribuyeron al choque cultural académico fueron esa falta de interacción entre profesorado y alumnado, es mucho menor la retroalimentación por parte del docente y también su escasa involucración en el "éxito" académico del alumno. 
Raluy (2012) ha explicado las diferencias semánticas entre el uso de la palabra "éxito" en español y en inglés, así como en ambos contextos culturales (España y EE. UU.). Ello podría implicar un malentendido entre idiomas y culturas: "Mientras presumimos que muchos de los alumnos estadounidenses definen el 'éxito académico' como la consecución de una matrícula de honor (o una nota muy alta) [...], imaginamos que el docente español lo define como la transmisión de conocimientos a su alumnado sin necesidad de interferir en el proceso y sin conceder mayor importancia a la nota que consigan (ya que no existe tanta obsesión con las notas en la sociedad española como en los EE. UU.)" (Pandor, 2017, p. 198).

Después de cuanto hemos dicho, y a la luz de los resultados de nuestro estudio, podemos concluir que la mayoría de las dificultades de la experiencia de matrícula directa tiene que ver, por tanto, con las diferencias respecto a la cultura académica española y la falta de conocimientos previos sobre ella. Consecuentemente, se confirma la hipótesis que planteábamos al inicio acerca de que existe un choque grave de cultura académica, por lo que consideramos que las contribuciones de nuestro estudio podrían resultar de interés, ya que a partir de aquí puede explorarse el modo de preparar al estudiante para que se adapte mejor al enfoque académico de cada sistema universitario (en ambos sentidos). Como decíamos, y de manera muy general, en los EE. UU., este se centra en el alumno (que a menudo es considerado una especie de cliente, puesto que paga una matrícula sustanciosa para asistir a la universidad), mientras que en España (al menos por ahora, pues es cierto que también se está caminando en esa dirección) se le da más importancia a la investigación de los docentes que a su actuación en el aula, al tiempo que existe la percepción de que el alumno ha de ser más autónomo. Ello provoca que el alumnado estadounidense se enfrente a una realidad dura por inesperada cuando llega a una universidad extranjera pensando que su rendimiento académico y satisfacción personal van a seguir siendo la única prioridad de sus profesores en el nuevo contexto.

Como todo trabajo de estas características, nuestro estudio presenta sin duda ciertas limitaciones al tratarse de una investigación cualitativa con hallazgos que se basan en las percepciones de nuestros participantes. También nos vimos limitados por el número final de participantes (95), que siempre es mejor cuanto más amplio. Y, por último, al tratarse de encuesta en línea, a través de la que inevitablemente se pierde un elemento de contacto humano que podrían aportar las entrevistas personales. No obstante, creemos que esta investigación aporta datos novedosos sobre las percepciones de este alumnado y, sobre todo, abre un ámbito de estudio que ha sido totalmente inexplorado hasta ahora.

En este sentido, entre las futuras líneas de investigación proponemos indagar en la percepción de este contraste desde la perspectiva del profesorado universitario español. Esto les haría ser más conscientes del choque cultural académico existente y de las formas en que podría atajarse. También serviría con el fin de diseñar materiales para el colectivo de estudiantes de Study Abroad con matrícula 
directa que les prepararan para esta estancia académica. A su vez, nuestro estudio abre posibilidades para investigar sobre la preparación aportada en la universidad de origen y de acogida para mejorar la orientación recibida por el alumnado antes de su estancia y durante ella. Con todo ello esperamos contribuir a un mejor desarrollo de la actual internacionalización de los estudios universitarios que no pueden ser sino multilingües y multiculturales.

\section{REFERENCIAS}

Abad, Alfonso. 2008. A study of the effect of study abroad and the homestay on the development of linguistic and interactional practices by Spanish L2 learners. Tesis de doctorado. Texas, Estados Unidos: Universidad de Texas.

Ainciburu, Cecilia. 2012. Planificación, interacción y aprendizaje cooperativo en un campus virtual: un curso de español académico ELE. En Hernández, C. (ed.). La Red y sus aplicaciones en la enseñanza-aprendizaje del español como lengua extranjera, XXII Congreso Internacional ASELE, Universidad de Valladolid, España.

Ainciburu, Cecilia. 2013. Modelos y recursos de escritura académica en diferentes lenguas maternas y su influencia en la práctica ELE. En Blecua, B., Borell, S., Crous, B. y Sierra, F. (eds.). Plurilingüismo y enseñanza de ELE en contextos multiculturales. XXIII Congreso Internacional ASELE, Universitat de Girona.

Ainciburu, Cecilia y Rodríguez, Patricia. 2015. La elección de géneros textuales en los exámenes de certificación de lenguas académicas. Las necesidades del alumno universitario y la evaluación implícita de competencias no lingüísticas. En Morimoto, Y., Pavón, Ma V. y Santamaría, R. (eds.). La enseñanza de ELE centrada en el alumno, XXV Congreso Internacional ASELE, Universidad Carlos III.

Ballano, Inmaculada y Muñoz, Itziar. 2015. La escritura académica en las universidades españolas. Maior Bilduma, 20.

Bazerman, Charles, Little, Joseph, Bethel, Lisa, Chavkin, Theri, Fouquette, Danielle y Garufis, Janet. 2016. Escribir a través del currículum. Guía de referencia. Córdoba: Universidad de Córdoba.

Bolívar, Adriana y Parodi, Giovanni. 2015. Academic and Professional Discourse. En Lacorte, Manel (ed.). The Routledge Handbook of Hispanic Applied Linguistics. New York: Routledge, 459-476.

Bonilla, Nancy. 2015. Fostering Students' Proficiency During Study Abroad: A Mixed-Methods Investigation of One Program's Response. Tesis de doctorado. Disponible en https://goo.gl/5jqxkx [Consulta: 14/9/17].

Castelló, Montserrat. 2015. Writing at the University. The Perspectives of the Stakeholders: Students and Faculty / no monográfico: Escribir en la univer- 
sidad. La perspectiva de los protagonistas: estudiantes y profesores. Cultura y Educación: Culture and Education, Volume 27, Issue 3.

Cohen, Andrew, Paige, R. Michael, Kappler, Barbara, Chi, Juli C. y Lassegard, James P. Shively. 2005. Maximizing study abroad: A students' guide to strategies for language and culture learning and use. Minneapolis, MN: Center for Advanced Research on Language Acquisition.

González, Maite. 2004. Las dificultades culturales y las diferencias educativas en los programas académicos de EE. UU. en España. En E. Martinell (coord.), La oferta formativa del profesorado de E/LE. Madrid: Edinumen, pp. 61-74.

González, Vicenta. 2001. Modificaciones en el discurso del profesor de español como lengua extranjera. Tesis de doctorado. Barcelona: Universidad de Barcelona.

Griffin, Kim. 2007. El pasaporte europeo lingüístico: Evaluación del nivel de competencia oral en español de anglohablantes que estudian en España. En E. Balmaseda Maestu (ed.). Las destrezas orales en la enseñanza del español L2-LE (Vol. 1). Logroño: Universidad de la Rioja, pp. 113-129.

(IIE) Institute of International Education. 2016. Press Release: Open Doors 2016 Executive Summary. Disponible en https:/www.iie.org/en/Why-IIE/ Announcements/2016-11-14-Open-Doors-Executive-Summary [Consulta: 14/9/17].

Jiménez, Francisco y López, Ismael. (ed.). 2017. Metodología para el trabajo con géneros discursivos académicos. Cáceres: Universidad de Extremadura.

Kinginger, Celeste, Wu, Qian, Lee, Sheng-Hsun y Tan, Dali. 2016. The shortterm homestay as a context for language learning. En H. Martin (Ed.), Study Abroad Research in Second Language Acquisition and International Education, 1(1), 34-60. Amsterdam: John Benjamins.

Lacorte, Manel y Suárez, Jesús. 2014. Teaching and Learning Spanish in the United States in the 21st Century / La enseñanza y el aprendizaje del español en los Estados Unidos en el siglo XXI. Journal of Spanish Language Teaching, 1:2. Special Issue.

MCERL. 2002. Marco común europeo de referencia para las lenguas: aprendizaje, enseñanza, evaluación. Madrid: Ministerio de Educación, Cultura y Deporte.

Mendoza, Arturo. 2015. La selección de tareas de escritura en los exámenes de ELE para el ámbito académico. Revista Nebrija de Lingüistica aplicada, 18 (9). Morell, Teresa. 2004. La interacción en la clase magistral. Alicante: Universidad de Alicante.

Morell, Teresa. 2009. ¿Cómo podemos fomentar la participación en nuestras clases universitarias? Alcoy: Marfil/Universidad de Alicante/Instituto de Ciencias de la Educación.

Navarro, Federico, Ávila Reyes, Natalia, Tapia-Ladino, Mónica, Cristovaö, Vera, Moritz, María Ester, Narváez Cardona, Elizabeth y Bazerman, Charles. 2016. 
Panorama histórico y contrastivo de los estudios sobre lectura y escritura en educación superior publicados en América Latina. Revista Signos 49 (1), 100126. Número especial monográfico "Procesos de lectura y escritura en el mundo contemporáneo de lengua española”

Ortega, Jenaro. 2001. La lección magistral. En G. Vázquez (Coord.), El discurso académico oral: Guía didáctica para la comprensión auditiva y visual de clases magistrales. Madrid: Edinumen, pp. 15-42.

Pandor, Jillian. 2017. Integración académica del alumnado estadounidense en la universidad española: el choque lingüístico y cultural. Tesis doctoral inédita. Alicante: Universidad de Alicante.

Pastor Cesteros, Susana. 2006. La enseñanza del español como lengua vehicular en contextos académicos. Marcoele, 2.

Pastor Cesteros, Susana. 2014. Aprendizaje por contenidos a través del Español como Segunda Lengua en la Educación Superior (ESLES): un puente entre la lengua y el conocimiento. E-Journal (EuroAmerican Journal) of Applied Linguistics and Languages, 1 (1), 15-30.

Pastor Cesteros, Susana. 2015a. Investigación en docencia por contenidos (español como segunda lengua) en el proceso de internacionalización de la Universidad de Alicante. En Álvarez, J.D., Tortosa, M.T., y Pellín, N. (coords.). Investigación y propuestas innovadoras de Redes UA para la mejora docente. Alicante: ICE Universidad de Alicante, cap. 73, pp. 1376-1400.

Pastor Cesteros, Susana. 2015b. Guía académica para estudiantes internacionales. Alicante: Universidad de Alicante.

Pastor Cesteros, Susana. 2016. Español académico e inmersión universitaria del alumnado extranjero de la Universidad de Alicante. En Álvarez, J.D., Tortosa, M.T., y Pellín, N. (coords.). Innovaciones metodológicas en docencia universitaria: resultados de investigación. Alicante: ICE Universidad de Alicante, 17371749.

Pastor Cesteros, Susana y Rodríguez-Lifante, Alberto. 2017. Español académico a través de la Guía académica para estudiantes internacionales de la Universidad de Alicante. e-AESLA, 3.

Pérez-Bedmar, Mónica. 2015. Intercultural Competence: Nature vs. Nuture. Ponencia presentada en II Jornadas de CIEE Alicante. Soñar en español: Desarrollo de la competencia lingüística e intercultural en programas de inmersión en el extranjero. Alicante: Universidad de Alicante.

Raluy, Ángel. 2012. El concepto estadounidense de “éxito" frente a su homónimo español: dos visiones sociológica, semántica y etimológicamente diferentes. ELUA: Estudios de Lingüistica de la Universidad de Alicante, 26, p. 269-288.

Regueiro, María Luisa y Sáez, Daniel. 2013. El español académico. Guía práctica para la elaboración de textos académicos. Madrid: Arco.

Reinders, Hayo, Moore, Nick y Lewis, Marilyn. 2008. The International Student 
Handbook. London: Palgrave.

Reinders, Hayo, Lewis, Marilyn y Phung, Linh. 2017. Studying in English. Strategies for Success in Higher Education. London: Palgrave. 2nd ed.

Rueda, Mercedes. 2006. Dos mundos distintos: La integración de alumnos estadounidenses en la vida familiar, universitaria y social española. En A. Álvarez, L. Barrientos, M. Braña, V. Coto, M. Cuevas, C. de la Hoz, I. Iglesias, P. Martínez, M. Prieto \& A. Turza (Eds.). La Competencia Pragmática y la Enseñanza del Español como Lengua Extranjera, pp. 557-568. Oviedo: Universidad de Oviedo.

Segalowitz, Norman, Freed, Barbara, Collentine, Joe, Lafford, Barbara, Lazar, Nicoles y Díaz-Campos, Manuel. 2004. A comparison of Spanish second language acquisition in two different learning contexts: Study abroad and the domestic classroom. Frontiers: The Interdisciplinary Journal of Study Abroad, 10, $1-18$

Vázquez, Graciela. 2001a. Guía didáctica del discurso académico escrito. ¿Cómo se escribe una monografía?. Madrid: Edinumen.

Vázquez, Graciela. 2001b. Actividades para la escritura académica. Madrid: Edinumen.

Vázquez, Graciela. 2001c. El discurso académico oral. Guía didáctica para la comprensión auditiva y visual de clases magistrales. Madrid: Edinumen.

Vázquez, Graciela. 2001d. Comprender en español. CD-Rom interactivo. Madrid: Edinumen.

Vázquez, Graciela. 2001e. El discurso académico español: cuestiones preliminares de didactización a través de materiales multimedia y unidades didácticas para el autoaprendizaje. En Pastor Cesteros, Susana y Salazar, Ventura (eds.). Tendencias y lineas de investigación en Adquisición de Segundas Lenguas. Alicante: Universidad de Alicante, pp. 337-348.

Vázquez, Graciela. 2004. La enseñanza del español con fines académicos. En Jesús Sánchez Lobato e Isabel Santos Gargallo (eds.). Vademécum para la formación de profesores. Enseñar español como segunda lengua y lengua extranjera. Madrid: SGEL, pp. 1129-1147.

Vázquez, Graciela. 2005. Español con fines académicos: de la comprensión a la producción de textos. Madrid: Edinumen.

Vázquez, Graciela. 2009. El discurso académico español: entre la identidad académica y la globalización del conocimiento. En Fernández, I. y Vera, A. (eds.). El español en contextos específicos: enseñanza e investigación. Santander: ASELE/ Fundación Comillas, pp. 117-128. 